\title{
The isomorphism problem for group algebras: A criterion
}

\author{
Taro Sakurai \\ Communicated by Britta Spaeth
}

\begin{abstract}
Let $R$ be a finite unital commutative ring. We introduce a new class of finite groups, which we call hereditary groups over $R$. Our main result states that if $G$ is a hereditary group over $R$, then a unital algebra isomorphism between group algebras $R G \cong R H$ implies a group isomorphism $G \cong H$ for every finite group $H$. As application, we study the modular isomorphism problem, which is the isomorphism problem for finite $p$-groups over $R=\mathbb{F}_{p}$, where $\mathbb{F}_{p}$ is the field of $p$ elements. We prove that a finite $p$-group $G$ is a hereditary group over $\mathbb{F}_{p}$ provided $G$ is abelian, $G$ is of class two and exponent $p$, or $G$ is of class two and exponent four. These yield new proofs for the theorems by Deskins and Passi-Sehgal.
\end{abstract}

\section{Introduction}

Let $R$ be a unital commutative ring (throughout this paper, we do not impose a ring (or algebra) to have 1 , but we impose $0 \neq 1$ if it has 1 ) and $G$ a finite group. The structure of the group algebra $R G$ of $G$ over $R$ reflects the structure of the group $G$ to some extent. The isomorphism problem asks whether a group algebra $R G$ determines the group $G$. See a survey by Sandling [20] for a historical account for this problem. In what follows, $p$ stands for a prime number, and $\mathbb{F}_{p}$ denotes the field of $p$ elements. The only classic isomorphism problem that is still open for more than 60 years is the modular isomorphism problem.

Problem. Let $G$ and $H$ be finite $p$-groups. Does a unital algebra isomorphism $\mathbb{F}_{p} G \cong \mathbb{F}_{p} H$ imply a group isomorphism $G \cong H$ ?

Several positive solutions for special classes of $p$-groups are known; see lists in the introduction of [6] or [8].

In this paper, we introduce a new class of finite groups, which we call hereditary groups over a finite unital commutative ring $R$ (Definition 1.4). Our main result (Criterion 1.6) states that if $G$ is a hereditary group over $R$, then a unital algebra isomorphism $R G \cong R H$ implies a group isomorphism $G \cong H$ for every finite group $H$. The proof rests on counting homomorphisms (Lemma 1.2) and 
adjoint (Lemma 1.3) - this indirect approach is novel in the sense that it does not involve normalized isomorphisms, for example. As application, we study the modular isomorphism problem. We prove that a finite $p$-group $G$ is a hereditary group over $\mathbb{F}_{p}$ provided $G$ is abelian (Lemma 3.2), $G$ is of class two and exponent $p$ (Lemma 3.5), or $G$ is of class two and exponent four (Lemma 3.10). These yield new proofs for the theorems by Deskins (Theorem 3.1) and Passi-Sehgal (Theorems 3.4 and 3.7) which are early theorems on the modular isomorphism problem.

\section{Criterion}

This section is devoted to proving our main result (Criterion 1.6). The first lemma is an easy application of the inclusion-exclusion principle.

Lemma 1.1. Let $G$ and $H$ be finite groups. We denote by $\operatorname{Epi}(G, H)$ the set of all epimorphisms from $G$ to $H$. Let $\mathscr{H}$ be the set of all maximal subgroups of $H$. Then

$$
|\operatorname{Epi}(G, H)|=\sum_{\mathcal{K} \subseteq \mathscr{H}}(-1)^{|\mathcal{K}|}\left|\operatorname{Hom}\left(G, \bigcap_{K \in \mathcal{K}} K\right)\right| .
$$

Proof. First, note that

$$
\begin{aligned}
\operatorname{Epi}(G, H) & =\{f: G \rightarrow H \mid \operatorname{im} f=H\} \\
& =\bigcap_{K \in \mathscr{H}}\{f: G \rightarrow H \mid \operatorname{im} f \not \leq K\} .
\end{aligned}
$$

Thus, by letting $\operatorname{Hom}^{K}(G, H)=\{f: G \rightarrow H \mid \operatorname{im} f \leq K\}$, it becomes

$$
\operatorname{Epi}(G, H)=\operatorname{Hom}(G, H)-\bigcup_{K \in \mathscr{H}} \operatorname{Hom}^{K}(G, H) .
$$

By the inclusion-exclusion principle, we have

$$
\begin{aligned}
|\operatorname{Epi}(G, H)| & =|\operatorname{Hom}(G, H)|-\left|\bigcup_{K \in \mathscr{H}} \operatorname{Hom}^{K}(G, H)\right| \\
& =|\operatorname{Hom}(G, H)|+\sum_{\emptyset \neq \mathcal{K} \subseteq \mathscr{H}}(-1)^{|\mathcal{K}|}\left|\bigcap_{K \in \mathcal{K}} \operatorname{Hom}^{K}(G, H)\right| \\
& =|\operatorname{Hom}(G, H)|+\sum_{\emptyset \neq \mathcal{K} \subseteq \mathscr{H}}(-1)^{|\mathcal{K}|}\left|\operatorname{Hom}\left(G, \bigcap_{K \in \mathcal{K}} K\right)\right| \\
& =\sum_{\mathcal{K} \subseteq \mathscr{H}}(-1)^{|\mathcal{K}|}\left|\operatorname{Hom}\left(G, \bigcap_{K \in \mathcal{K}} K\right)\right| .
\end{aligned}
$$

(Note that if $\mathcal{K}=\emptyset$, then $\bigcap_{K \in \mathcal{K}} K=\{h \in H \mid h \in K(K \in \mathcal{K})\}=H$.) 
The next lemma is inspired by the work of Lovász $[11,12]$.

Lemma 1.2. Let $G$ and $H$ be finite groups. Then $G \cong H$ if and only if $|G|=|H|$ and

$$
|\operatorname{Hom}(G, K)|=|\operatorname{Hom}(H, K)|
$$

for every subgroup $K$ of $G$.

Proof. From $|\operatorname{Hom}(G, K)|=|\operatorname{Hom}(H, K)|$ for every subgroup $K$ of $G$, it follows that $|\operatorname{Epi}(H, G)|=|\operatorname{Epi}(G, G)| \geq 1$ by Lemma 1.1. Hence, we have an epimorphism from $H$ to $G$. It must be an isomorphism because $|G|=|H|$.

For a unital $R$-algebra $A$ over a unital commutative ring $R$, the unit group of $A$ is denoted by $A^{*}$. The following adjoint, which rephrases the universal property of group algebras [15, Proposition 3.2.7], is well-known.

Lemma 1.3. Let $R$ be a unital commutative ring, $G$ a group and $A$ a unital $R$-algebra. Then there is a bijection

$$
\operatorname{Hom}(R G, A) \cong \operatorname{Hom}\left(G, A^{*}\right)
$$

which is natural in $G$ and A. (Namely, the group algebra functor is left adjoint to the unit group functor.)

Proof. The restriction $(f: R G \rightarrow A) \mapsto\left(\left.f\right|_{G}: G \rightarrow A^{*}\right)$ gives rise to the desired bijection; see [17, pp. 204-205, 490] for details.

Now we propose a definition of hereditary groups which is crucial in this study.

Definition 1.4. Set $M=\{[G] \mid G$ is a finite group $\}$, where the symbol $[G]$ denotes the isomorphism class of a group $G$. It becomes a commutative monoid with an operation $[G]+[H]=[G \times H]$. Let $K(M)$ denote the Grothendieck group ${ }^{1}$ of $M$. As it is a $\mathbb{Z}$-module (abelian group), we can extend scalars and obtain a $\mathbb{Q}$-vector space $L(M)=\mathbb{Q} \otimes_{\mathbb{Z}} K(M)$. For a finite unital commutative ring $R$, define the $\mathbb{Q}$-subspace $S(R)$ of $L(M)$ by

$$
S(R)=\sum_{\substack{A \text { is a finite } \\ \text { unital } R \text {-algebra }}} \mathbb{Q}\left[A^{*}\right] .
$$

Namely, $S(R)$ is the $\mathbb{Q}$-subspace of $L(M)$ spanned by the isomorphism classes of unit groups of finite unital $R$-algebras. We call a finite group $G$ a hereditary group over $R$ if $[K] \in S(R)$ for every subgroup $K$ of $G$.

\footnotetext{
1 This is also called the group completion of $M$. See [16, Theorem 1.1.3].
} 
From the definition, being hereditary group is a subgroup-closed property. Note that the group completion $M \rightarrow K(M)$ is injective because $M$ is cancellative by the Krull-Schmidt theorem; the localization $K(M) \rightarrow L(M)$ is also injective because $K(M)$ is torsion-free. Thus, $M$ can be identified with a submonoid of $L(M)$.

Example 1.5. Let $C_{q}$ denote the cyclic group of order $q$. Then, from $\mathbb{F}_{5}^{*} \cong C_{4}$ and $\left(\mathbb{F}_{5} C_{5}\right)^{*} \cong C_{4} \times\left(C_{5}\right)^{4}$, we have

$$
\left[C_{5}\right]=\frac{1}{4}\left[\left(\mathbb{F}_{5} C_{5}\right)^{*}\right]-\frac{1}{4}\left[\mathbb{F}_{5}^{*}\right] \in S\left(\mathbb{F}_{5}\right) .
$$

In particular, $C_{5}$ is a hereditary group over $\mathbb{F}_{5}$.

The next criterion - our main result - shows that hereditary groups are determined by their group algebras.

Criterion 1.6. Let $G$ and $H$ be finite groups, and let $R$ be a finite unital commutative ring. Suppose $G$ is a hereditary group over $R$. If $R G \cong R H$, then $G \cong H$.

The proof is done by describing the number of group homomorphisms in terms of the number of unital algebra homomorphisms.

Proof of Criterion 1.6. Since a unital commutative ring $R$ has invariant basis number (IBN) property, we have $|G|=|H|$ from $R G \cong R H$. Hence, by Lemma 1.2, it suffices to prove that $|\operatorname{Hom}(G, K)|=|\operatorname{Hom}(H, K)|$ for every subgroup $K$ of $G$.

Since $G$ is a hereditary group over $R$, we have $[K] \in S(R)$. Therefore, there is a positive integer $n$ and finite unital $R$-algebras $A, B$ such that

$$
[K]=\frac{1}{n}\left[A^{*}\right]-\frac{1}{n}\left[B^{*}\right] .
$$

Namely, $A^{*} \cong B^{*} \times K^{n}$. Hence,

$$
\left|\operatorname{Hom}\left(G, A^{*}\right)\right|=\left|\operatorname{Hom}\left(G, B^{*} \times K^{n}\right)\right|=\left|\operatorname{Hom}\left(G, B^{*}\right)\right| \times|\operatorname{Hom}(G, K)|^{n} .
$$

Thus, by Lemma 1.3, we can obtain

$$
|\operatorname{Hom}(G, K)|=\left(\frac{\left|\operatorname{Hom}\left(G, A^{*}\right)\right|}{\left|\operatorname{Hom}\left(G, B^{*}\right)\right|}\right)^{1 / n}=\left(\frac{|\operatorname{Hom}(R G, A)|}{|\operatorname{Hom}(R G, B)|}\right)^{1 / n} .
$$

We can calculate $|\operatorname{Hom}(H, K)|$ similarly and conclude that

$$
|\operatorname{Hom}(G, K)|=|\operatorname{Hom}(H, K)|
$$

from $R G \cong R H$. 
Remark 1.7. Studying a finite group that is a "linear combination" of unit groups, precisely an element of $S(R)$, is essential because even the cyclic group of order five cannot be realized as a unit group of any unital ring. (See a theorem by Davis and Occhipinti [4, Corollary 3], for example.) This is quite different from the fact that every finite abelian $p$-group is a hereditary group over $\mathbb{F}_{p}$ (Lemma 3.2).

It also should be noted that no examples of non-hereditary groups are hitherto found.

Using this criterion, we provide new proofs for some early theorems on the modular isomorphism problem in the last section.

\section{Quasi-regular groups}

We show that, with Criterion 1.6, study of quasi-regular groups can be used to study the isomorphism problem. Throughout this section, $R$ denotes a unital commutative ring.

Definition 2.1. Let $A$ be an $R$-algebra. Define the quasi-multiplication on $A$ by

$$
x \circ y=x+y+x y .
$$

An element $x \in A$ is called quasi-regular if there is an element $y \in A$ such that $x \circ y=0=y \circ x$. We denote the set of all quasi-regular elements by $Q(A)$. It forms a group under the quasi-multiplication, and we call it the quasi-regular group of $A$. If $A=Q(A)$, then $A$ is called quasi-regular (or radical).

Quasi-multiplication is also called circle operation or adjoint operation. Accordingly, quasi-regular groups are also called circle groups or adjoint groups. As these terms have completely different meaning in other contexts, we avoid using them.

If an $R$-algebra $A$ has a multiplicative identity, then there is an isomorphism $Q(A) \rightarrow A^{*}$ that is defined by $x \mapsto 1+x$. We study how quasi-regular groups are related to unit groups, especially when algebras do not have multiplicative identities, in the rest of this section.

Definition 2.2. Let $A$ be an $R$-algebra. We denote the unitization of $A$ by $A_{\text {un }}$ : it is a direct product $A_{\text {un }}=A \times R$ as $R$-modules, and its multiplication is defined by

$$
(x, r) \times(y, s)=(s x+r y+x y, r s) .
$$

Note that $(0,1) \in A_{\text {un }}$ is the multiplicative identity. The unitization is also called the Dorroh extension, especially the case $R=\mathbb{Z}$. 
Lemma 2.3. Let $A$ be a quasi-regular $R$-algebra. Then an element $(x, r) \in A_{\text {un }}$ is a unit if and only if $r \in R$ is a unit.

Proof. The "only if" part is trivial. Let us assume $r \in R^{*}$ to show $(x, r) \in A_{\mathrm{un}}^{*}$. As $r^{-1} \in R$ exists and $r^{-1} x \in A$, there is an element $y \in A$ such that

$$
\left(r^{-1} x\right) \circ y=0=y \circ\left(r^{-1} x\right)
$$

because $A$ is quasi-regular. Then it can be shown that $(x, r)^{-1}=\left(r^{-1} y, r^{-1}\right)$ by direct calculation.

Lemma 2.4. Let $A$ be a quasi-regular $R$-algebra. Then $A_{\mathrm{un}}^{*} \cong Q(A) \times R^{*}$. In particular, $[Q(A)] \in S(R)$ if $R$ and $A$ are finite.

Proof. Note that there are homomorphisms

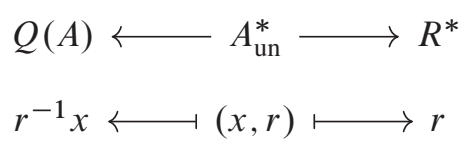

which are well-defined by Lemma 2.3. It is straightforward to check that these satisfy the universal property of a direct product.

Remark 2.5. For determining whether a finite group is a quasi-regular group of an $R$-algebra, a theorem by Sandling [18, Theorem 1.7] would be helpful. It should be noted that quasi-regular groups also have severely restricted structure as unit groups. See [19, p. 343].

\section{Modular isomorphism problem}

As application of Criterion 1.6, we provide new proofs for theorems by Deskins [5] and Passi-Sehgal [13] which are early theorems on the modular isomorphism problem.

\subsection{Abelian (class at most one)}

Let us state a well-known theorem by Deskins [5, Theorem 2].

Theorem 3.1 (Deskins). Let $G$ and $H$ be finite p-groups. Suppose $G$ is abelian. If $\mathbb{F}_{p} G \cong \mathbb{F}_{p} H$, then $G \cong H$.

To use our criterion, we need to prove the following, which is also useful to prove Theorems 3.4 and 3.7 . 
Lemma 3.2. Every finite abelian p-group is a hereditary group over $\mathbb{F}_{p}$.

Proof. Let $C_{p^{n}}$ be the cyclic $p$-group of order $p^{n}$. Since a finite abelian $p$-group is a direct product of finite cyclic $p$-groups and being finite cyclic $p$-group is a subgroup-closed property, it suffices to prove that $\left[C_{p^{n}}\right] \in S\left(\mathbb{F}_{p}\right)$. We prove it by induction on $n$.

Base case $(n=0)$. Clear by Definition 1.4.

Inductive case $(n>0)$. Let $\Delta\left(C_{p^{n}}\right)$ denote the augmentation ideal of $\mathbb{F}_{p} C_{p^{n}}$. Then

$$
\left(\mathbb{F}_{p} C_{p^{n}}\right)^{*}=V \times \mathbb{F}_{p}^{*},
$$

where $V=1+\Delta\left(C_{p^{n}}\right)$. It is clear that $V$ is a finite abelian group. Furthermore, it is easy to see the exponent of $V$ equals $p^{n}$. Therefore, $V \cong \prod_{i=1}^{n}\left(C_{p^{i}}\right)^{a_{i}}$ for some non-negative integers $a_{1}, \ldots, a_{n-1}$ and a positive integer $a_{n}$. Hence,

$$
\begin{aligned}
{\left[C_{p^{n}}\right] } & =\frac{1}{a_{n}}[V]-\sum_{i=1}^{n-1} \frac{a_{i}}{a_{n}}\left[C_{p^{i}}\right] \\
& =\frac{1}{a_{n}}\left[\left(\mathbb{F}_{p} C_{p^{n}}\right)^{*}\right]-\frac{1}{a_{n}}\left[\mathbb{F}_{p}^{*}\right]-\sum_{i=1}^{n-1} \frac{a_{i}}{a_{n}}\left[C_{p^{i}}\right],
\end{aligned}
$$

and we have $\left[C_{p^{n}}\right] \in S\left(\mathbb{F}_{p}\right)$ by induction. (This lemma can be also proved by appealing to the structure theorem of $\left(\mathbb{F}_{p} C_{p^{n}}\right)^{*}$ by Janusz [9, Theorem 3.1].)

With this lemma, we provide a new proof of the Deskins theorem.

Proof of Theorem 3.1. Since the finite abelian $p$-group $G$ is a hereditary group over $\mathbb{F}_{p}$ by Lemma 3.2, $\mathbb{F}_{p} G \cong \mathbb{F}_{p} H$ implies $G \cong H$ by Criterion 1.6.

Remark 3.3. Besides the original proof by Deskins, an alternative simple proof is given by Coleman [3, Theorem 4]. Proofs can be found in monographs such as [10, Theorem 2.4.3], [14, Lemma 14.2.7], [22, (III.6.2)], [15, Theorem 9.6.1], or [23, Theorem 4.10] as well.

\subsection{Class two and exponent $p$}

The aim of this subsection is to provide a new proof of the following theorem by Passi and Sehgal [13, Corollary 13].

Theorem 3.4 (Passi-Sehgal). Let $G$ and $H$ be finite p-groups. Suppose $G$ is of class two and exponent $p$. If $\mathbb{F}_{p} G \cong \mathbb{F}_{p} H$, then $G \cong H$.

See also Remark 3.8. To use our criterion, we need to prove the following. 
Lemma 3.5. Every finite $p$-group of class two and exponent $p$ is a hereditary group over $\mathbb{F}_{p}$.

A key ingredient for the proof is a slight modification of the theorem by Ault and Watters $[1,7]$.

Theorem 3.6 (Ault-Watters). Let $G$ be a finite p-group. Suppose $G$ is of class two and exponent $p$. Then there is a finite quasi-regular $\mathbb{F}_{p}$-algebra $A$ with $Q(A) \cong G$.

Proof. It is proved in [1] that there is a finite quasi-regular ring $A=G$ with operations $^{2}$ defined by

$$
\begin{aligned}
g+h & =g \cdot h \cdot m(g, h)^{-1}, \\
g \times h & =m(g, h)
\end{aligned}
$$

and $Q(A) \cong G$; here $m: G \times G \rightarrow \zeta(G)$ is a certain map, where $\zeta(G)$ is the center of $G$. In particular, $1 \in G$ is the additive identity of $A$. By induction, it can be shown that

$$
\underbrace{g+\cdots+g}_{n}=g^{n} \cdot m(g, g)^{-n(n-1) / 2}
$$

for a positive integer $n$. Since the prime $p$ is odd because a group of exponent two cannot be of class two, we can prove

$$
\underbrace{g+\cdots+g}_{p}=1
$$

Therefore, there is a canonical $\mathbb{F}_{p}$-algebra structure on $A$.

Proof of Lemma 3.5. Since finite abelian $p$-groups are hereditary groups over $\mathbb{F}_{p}$ by Lemma 3.2, it suffices to prove that $[G] \in S\left(\mathbb{F}_{p}\right)$ for every finite $p$-group $G$ of class two and exponent $p$. Because such $p$-group $G$ is a quasi-regular group of some finite quasi-regular $\mathbb{F}_{p}$-algebra by Theorem 3.6, $[G] \in S\left(\mathbb{F}_{p}\right)$ follows from Lemma 2.4.

Now we are in position to prove the theorem by Passi and Sehgal.

Proof of Theorem 3.4. Since the finite $p$-group $G$ of class two and exponent $p$ is a hereditary group over $\mathbb{F}_{p}$ by Lemma $3.5, \mathbb{F}_{p} G \cong \mathbb{F}_{p} H$ implies $G \cong H$ by Criterion 1.6.

\footnotetext{
${ }^{2}$ Beware that the operations defined in [1] are incorrect; it is corrected in [7].
} 


\subsection{Class two and exponent four}

The aim of this subsection is to prove the even prime counterpart of Theorem 3.4.

Theorem 3.7 (Passi-Sehgal). Let $G$ and $H$ be finite 2-groups. Suppose $G$ is of class two and exponent four. If $\mathbb{F}_{2} G \cong \mathbb{F}_{2} H$, then $G \cong H$.

Remark 3.8. Note that the third dimension subgroup of $G$ modulo $p$ is $G^{p} \gamma_{3}(G)$ if $p \neq 2$ and $G^{4}\left(G^{\prime}\right)^{2} \gamma_{3}(G)$ if $p=2$, where $\gamma_{3}(G)$ denotes the third term of the lower central series of $G$. Thus, the assumption for a group in Theorem 3.4 or Theorem 3.7 holds if $G$ has the trivial third modular dimension subgroup. Actually, this is how assumption is stated by Passi and Sehgal [13, Corollary 7].

Nowadays, more is known. A theorem by Sandling [21, Theorem 1.2] provides a positive solution for a finite $p$-group of class two with elementary abelian commutator subgroup.

A strategy for the proof is the same as Theorem 3.4. The even prime counterpart of the Ault-Watters theorem is the following theorem by Bovdi [2].

Theorem 3.9 (Bovdi). Let $G$ be a finite 2-group. Suppose $G$ is of class two and exponent four. Then there is a finite quasi-regular $\mathbb{F}_{2}$-algebra $A$ with $Q(A) \cong G$.

Lemma 3.10. Every 2-group of class two and exponent four is a hereditary group over $\mathbb{F}_{2}$.

Proof. As all finite abelian 2-groups are hereditary groups over $\mathbb{F}_{2}$ by Lemma 3.2, it suffices to prove that $[G] \in S\left(\mathbb{F}_{2}\right)$ for every finite 2-group $G$ of class two and exponent four. Because such 2-group $G$ is a quasi-regular group of some finite quasiregular $\mathbb{F}_{2}$-algebra by Theorem 3.9, $[G] \in S\left(\mathbb{F}_{2}\right)$ follows from Lemma 2.4.

Proof of Theorem 3.7. Since the finite 2-group $G$ of class two and exponent four is a hereditary group over $\mathbb{F}_{2}$ by Lemma $3.10, \mathbb{F}_{2} G \cong \mathbb{F}_{2} H$ implies $G \cong H$ by Criterion 1.6.

\section{Bibliography}

[1] J. C. Ault and J. F. Watters, Circle groups of nilpotent rings, Amer. Math. Monthly 80 (1973), 48-52.

[2] A. A. Bovdi, On circle groups of nilpotent rings of characteristic 2, Period. Math. Hungar. 32 (1996), 31-34.

[3] D. B. Coleman, On the modular group ring of a p-group, Proc. Amer. Math. Soc. 15 (1964), 511-514. 
[4] C. Davis and T. Occhipinti, Which finite simple groups are unit groups?, J. Pure Appl. Algebra 218 (2014), 743-744.

[5] W.E. Deskins, Finite abelian groups with isomorphic group algebras, Duke Math. J. 23 (1956), 35-40.

[6] B. Eick and A. Konovalov, The modular isomorphism problem for the groups of order 512, in: Groups St. Andrews 2009. Vol. 2, Cambridge University, Cambridge (2011), 375-383.

[7] R. Gilmer and D. Roselle, Complements and comments, Amer. Math. Monthly 80 (1973), 1116-1118.

[8] M. Hertweck and M. Soriano, On the modular isomorphism problem: groups of order $2^{6}$, in: Groups, Rings and Algebras, Contemp. Math. 420, American Mathematical Society, Providence (2006), 177-213.

[9] G. J. Janusz, Faithful representations of $p$ groups at characteristic p. I, J. Algebra 15 (1970), 335-351.

[10] R. L. Kruse and D. T. Price, Nilpotent Rings, Gordon and Breach Science, New York, 1969.

[11] L. Lovász, Operations with structures, Acta Math. Acad. Sci. Hung. 18 (1967), $321-328$.

[12] L. Lovász, Direct product in locally finite categories, Acta Sci. Math. (Szeged) 33 (1972), 319-322.

[13] I. B. S. Passi and S. K. Sehgal, Isomorphism of modular group algebras, Math. Z. 129 (1972), 65-73.

[14] D. S. Passman, The Algebraic Structure of Group Rings, Pure Appl. Math., John Wiley \& Sons, New York, 1977.

[15] C. Polcino Milies and S. K. Sehgal, An Introduction to Group Rings, Algebra and Applications. 1, Kluwer Academic, Dordrecht, 2002.

[16] J. Rosenberg, Algebraic K-theory and its Applications, Grad. Texts in Math. 147, Springer, New York, 1994.

[17] L. H. Rowen, Graduate Algebra: Noncommutative View, Grad. Stud. Math. 91, American Mathematical Society, Providence, 2008.

[18] R. Sandling, Group rings of circle and unit groups, Math. Z. 140 (1974), 195-202.

[19] R. Sandling, Units in the modular group algebra of a finite Abelian $p$-group, J. Pure Appl. Algebra 33 (1984), 337-346.

[20] R. Sandling, The isomorphism problem for group rings: a survey, in: Orders and Their Applications, Lecture Notes in Math. 1142, Springer, Berlin (1985), 256-288.

[21] R. Sandling, The modular group algebra of a central-elementary-by-abelian $p$-group, Arch. Math. (Basel) 52 (1989), 22-27. 
[22] S. K. Sehgal, Topics in Group Rings, Monogr. Textb. Pure Appl. Math. 50, Marcel Dekker, New York, 1978.

[23] S. K. Sehgal, Group rings, in: Handbook of Algebra. Vol. 3, Elsevier/North-Holland, Amsterdam (2003), 457-541.

Received May 16, 2019; revised November 19, 2019.

\section{Author information}

Taro Sakurai, Department of Mathematics and Informatics, Graduate School of Science, Chiba University, 1-33, Yayoi-cho, Inage-ku, Chiba-shi, Chiba, 263-8522, Japan.

E-mail: tsakurai@math.s.chiba-u.ac.jp 